\title{
Perfil nutricional e alimentar de escolares matriculados em uma escola municipal
}

\author{
Nutritional and food profile of schoolchildren enrolled in a municipal school
}

\author{
Joane Diomara Coleone ${ }^{a}$, Daiana Argenta Kümpelb, Ana Luisa Sant'Anna Alvesc, \\ Carolina Benvenuti de Mattos $^{d}$ \\ a Graduada em Nutrição pela Universidade de Passo Fundo. \\ b Nutricionista. Mestre em Envelhecimento Humano e docente do Curso de Nutrição da Universidade de Passo Fundo. \\ c Nutricionista. Doutora em Epidemiologia e docente do Curso de Nutrição da Universidade de Passo Fundo. \\ d Nutricionista. Mestre em Ciências Médicas: Endocrinologia e docente do curso de Nutrição da Universidade de Passo Fundo.
}

RESUMO Objetivo: Conhecer o perfil nutricional e alimentar de escolares matriculados na Escola Municipal de Ipiranga do Sul. Materiais e Métodos: Estudo transversal com 113 escolares de 5 a 19 anos, matriculados em uma Escola Municipal de Ipiranga do Sul (RS). Os dados foram coletados através de questionários e medidas antropométricas. As variáveis sociodemográficas investigadas foram: idade, sexo, cor, escolaridade, classe econômica e recebimento de benefício pelo Programa Bolsa Família (PBF). Avaliou-se o estado nutricional dos escolares através da classificação do índice de massa corporal para idade e circunferência do braço. Para a avaliação do consumo alimentar utilizou-se o questionário de frequência alimentar E-Kindex.

Resultados: Dos 113 escolares avaliados, prevaleceu o sexo masculino, crianças, cor de pele branca, pertencentes a classe econômica B e C e sem recebimento ao PBF. A maior prevalência foi de escolares eutróficos $(67,3 \%)$ e com consumo alimentar adequado. Na análise bivariada entre o estado nutricional e fatores associados, observa-se que a maior prevalência de sobrepeso/obesidade esteve associada a cor de pele não branca $(p=0,036)$, pertencer as classes econômicas C, D e E $(p=0,044)$ e ser beneficiado pelo PBF $(p=0,008)$.

Conclusão: A maioria dos escolares apresentaram-se eutróficos, porém 30,1\% estavam com excesso de peso. Destaca-se o baixo consumo diário de frutas, verduras e legumes, apesar da aparente adequação do consumo alimentar. Ações de educação alimentar e nutricional, previstas no Programa Nacional de Alimentação Escolar, devem ser reforçadas, a fim de promover melhora na situação alimentar e nutricional dos escolares.

Palavras-chave: transição nutricional; avaliação nutricional; consumo de alimentos; escolares.

ABSTRACT

Objective: To know the nutritional and food profile of schoolchildren enrolled in a Municipal School of Ipiranga do Sul.

Materials and Methods: Cross-sectional study with 113 schoolchildren from 5 to 19 years old, enrolled in a Municipal School of Ipiranga do Sul (RS). Data were collected through questionnaires and evaluation of anthropometric measures. The sociodemographic variables investigated were: age, sex, color, education, economic class and being part of the Bolsa Família Program (BFP). The nutritional state of schoolchildren was evaluated through the classification of the body mass index for age and the arm circumference. For the evaluation of food consumption, the E-Kindex frequency food questionnaire was used.

Results: Of the 113 schoolchildren evaluated, the most frequent were male sex, children, white skin color, belonging to economy classes $B$ and $C$ and without being in the BFP. The highest prevalence was of eutrophic children (67.3\%) and with adequate food consumption. In the bivariate analysis between nutritional state and associated factors, it was observed that the highest prevalence of overweight/obesity was associated with the non-white skin color $(p=0.036)$, belonging to economic classes $C, D$ and $E(p=0.044)$ and being part of the BFP $(p=0.008)$.

Conclusion: Most of the schoolchildren presented as eutrophic, although 30.1\% were overweight. Noteworthy is the low consumption of fruits and vegetables, despite the apparent adequacy of food consumption. Actions of food and nutritional education, provided in the National School Alimentation Program, should be strengthened, with the view to promote improvement in the food and nutritional state of schoolchildren.

Keywords: nutritional transition; nutritional evaluation; food consumption; schoolchildren. 


\section{INTRODUÇÃO}

A população brasileira vem passando por diversas alterações nos padrões dietéticos e nutricionais, nas diferentes classes sociais e faixas etárias, este processo é denominado transição nutricional, o qual é caracterizado pela redução das prevalências de déficits nutricionais e aumento do sobrepeso e obesidade ${ }^{1}$. Houve também mudanças na localização da prevalência da desnutrição, a qual antigamente era mais vista em regiões menos favorecidas, e o excesso de peso era mais visto em regiões mais ricas, processo este que não ocorre mais, pois hoje pode-se observar desnutrição e excesso de peso em qualquer região do mundo².

Muitos estudos mostram o aumento do excesso de peso em crianças e adolescentes na América e na Europa. No Brasil, no período entre 1974 e 2009, o excesso de peso entre adultos triplicou, representando $49 \%$ da população com sobrepeso e 14,6\% com obesidade. Deve-se dar ainda mais atenção ao fato de que o sobrepeso, entre as crianças é de $47,8 \%$ e nos adolescentes é de $21,5 \%{ }^{3}$. Os dados mais recentes adquiridos das Pesquisas de Orçamentos familiares (POF) de 2008/2009 evidenciam que o excesso de peso na adolescência, período compreendido entre 10 a 19 anos, passou de $16,6 \%$ para $21,7 \%$ no sexo masculino e de $15,1 \%$ para $19,4 \%$ para o sexo feminino 4 . Já na região sul brasileira, dentre as crianças de 5 a 9 anos, 2,5\% apresentam déficit de peso, $36,3 \%$ excesso de peso e $16,7 \%$ obesidade ${ }^{5}$.

$\mathrm{O}$ excesso de peso, principalmente em forma de gordura, aumenta a probabilidade de formação de diversas disfunções, bem como os índices de morbidade e mortalidade no mundo todo. Existem diversos fatores que, combinados ou isolados podem levar à obesidade, incluindo fatores ambientais, genéticos, fisiológicos, psíquicos e ambientais. Destaca-se como principal fator ambiental a grande ingestão de alimentos considerados inadequados para esta faixa etária, por possuírem alto valor calórico e baixo valor nutricional, associado ao sedentarismo, prática cada vez mais vista, gerando assim o aumento de peso em crianças e adolescentes ${ }^{6}$.

Para minimizar esta situação, a segunda Diretriz da Política Nacional de Alimentação e Nutrição, enfatiza a Promoção da Alimentação Adequada e Saudável. Sendo que deve estar de acordo com as necessidades de cada fase do curso da vida e incentivar a Educação Alimentar e Nutricional para a população ${ }^{7}$.

A infância e a adolescência são, portanto, as principais fases para determinação do perfil nutricional dos indivíduos, sendo este o melhor momento para se criar hábitos de vida saudáveis, fazendo com que as crianças cresçam com saúde, diminuindo assim, os gastos na saúde pública. No Brasil, programas como o Programa Nacional de Alimentação e Escolar podem contribuir para a melhoria do estado nutricional e consumo alimentar, uma vez que, a educação nutricional e o monitoramento do estado nutricional estão previstos. Desta forma, pode-se reduzir o risco para doenças crônicas não transmissíveis na infância e na vida adulta ${ }^{8,9}$.

Diante do exposto, este estudo tem como objetivo conhecer o perfil nutricional e alimentar de escolares matriculados em uma Escola Municipal de Ipiranga do Sul (RS).

\section{MATERIAIS E MÉTODOS}

Foi realizado um estudo transversal com escolares de idades entre 5 e 19 anos, matriculados na Escola Municipal Dom João Becker em Ipiranga do Sul no ano de 2015. A amostra avaliada foi constituída 113 escolares matriculados no ano de 2015, sendo excluídos aqueles com necessidades especiais que impossibilitassem a aferição completa das medidas antropométricas e a não participação no estudo.

Aplicou-se um questionário com variáveis sociodemográficas, a saber: cor da pele, idade e sexo. Para a classificação econômica, foi enviado aos pais ou responsáveis, o Critério de Classificação Econômica Brasil da ABEP (Associação Brasileira de Empresas e Pesquisas) ${ }^{10}$ de 2015, classificando-se em classe, A, B, C ou D-E. Também se avaliou o recebimento ou não de benefício pelo Programa Bolsa Família (PBF).

As medidas antropométricas foram aferidas pela acadêmica pesquisadora que recebeu treinamento específico para esta atividade e pela nutricionista do município. Para o cálculo do índice de massa corporal (IMC), foram avaliados peso e estatura, sendo que a classificação desta medida foi feita através das curvas de crescimento da Organização Mundial da Saúde11, a qual é recomendada para crianças e adolescentes de 5 a 19 anos.

Para aferir a circunferência do braço (CB) foi marcado um ponto médio entre o acrômio e o olecrano, com o braço não dominante em direção ao tórax, formando um ângulo de 90 o. O braço alongado foi contornado com a fita métrica flexível, de forma que fosse evitada a compressão da pele ou folga da mesma. Os resultados foram classificados segundo o percentual de adequação recomendado por Frisancho ${ }^{12}$.

Para avaliar o consumo alimentar aplicou-se o Questionário de Frequência Alimentar E-Kindex, que avalia hábitos alimentares, comportamentos e práticas que podem levar à obesidade em crianças. Para as crianças menores de 10 (dez) anos, este, foi respondido pelo responsável. Este questionário contém 13 alimentos que avaliam a frequência de ingestão de 11 grupos alimentares, assim como a técnica de cocção, frito ou grelhado. Os resultados variam de 0 a 37, sendo que, os alunos que apresentarem na soma do questionário a pontuação menor de 18 são classificados como tendo 
uma alimentação inadequada, já os alunos com pontuação maior de 19 são classificados como tendo uma alimentação adequada, desta forma, quanto mais alto for a pontuação, melhor é a qualidade da dieta ${ }^{13}$.

Os dados foram apresentados através de variáveis categóricas expressas em frequência absoluta e relativa simples. Para verificar a associação entre o desfecho e as variáveis de exposição foi aplicado o teste qui-quadrado. O nível de significância adotado foi de 95\%. Todas as análises foram realizadas utilizando-se o software SPSS versão 18.0.

Este estudo foi aprovado pelo Comitê de Ética em Pesquisa da Universidade de Passo Fundo CAAE: 44116015.0.0000.5342, sob parecer no 1.040.422, de acordo com a Resolução 466/12 do Conselho Nacional de Saúde, além disso todos os participantes da pesquisa foram preservados através do Termo de Consentimento Livre e Esclarecido.

\section{RESULTADOS}

Dos 113 escolares avaliados, prevaleceu o sexo masculino, crianças, cor de pele branca, pertencentes a classe econômica B e C e sem recebimento de benefício pelo PBF. Quanto ao estado nutricional e consumo alimentar, a maior prevalência foi de escolares eutróficos e com consumo alimentar adequado (Tabela 1).

$\mathrm{Na}$ análise bivariada entre o estado nutricional e fatores associados, observa-se que a maior prevalência de sobrepeso/obesidade esteve associada a cor de pele não branca $(p=0,036)$, pertencer as classes econômicas $C$, D e E $(p=0,044)$ e ser beneficiado pelo PBF $(p=0,008)$. Ser do sexo masculino e ter consumo alimentar inadequado também apresentaram maior prevalência de sobrepeso/obesidade, no entanto a associação não foi significativa (Tabela 2).
Quanto à frequência do consumo alimentar, observouse que o alimento mais consumido (mais de 6 vezes por semana) foi o leite, seguido de cereais e pães. Na frequência de 3 a 5 vezes na semana, os mais prevalentes são as carnes e frutas, já na frequência de 1 a 2 vezes na semana, destacase o refrigerante, seguido de alimentos fritos, grelhados, legumes, carnes defumadas, doces e petiscos, bem como as verduras e os legumes. O alimento que mais chama atenção na tabela é o peixe, o qual nunca é consumido pela maioria dos escolares, bem como as verduras e legumes que apresentaram baixo consumo (Tabela 3).

Tabela 1. Características sociodemográficas de crianças e adolescentes matriculados em escola pública de Ipiranga do Sul $(n=113)$.

\begin{tabular}{lcc}
\hline \multicolumn{1}{l}{ Variáveis } & Categoria & $\mathbf{n}(\mathbf{\%})$ \\
Sexo & Masculino & $58(51,3)$ \\
Idade & Feminino & $55(48,7)$ \\
& $5-9$ anos & $59(52,2)$ \\
Cor da pele & $10-14$ anos & $51(45,1)$ \\
& $14-19$ anos & $3(2,7)$ \\
Classe econômica & Branco & $101(89,4)$ \\
& Não branco & $12(10,7)$ \\
& A & $4(3,5)$ \\
Bolsa Família & B & $54(47,8)$ \\
& C & $48(42,5)$ \\
Consumo alimentar & D-E & $7(6,2)$ \\
& Sim & $12(10,6)$ \\
IMC/Idade & Não & $101(89,4)$ \\
& Inadequado & $30(26,5)$ \\
& Adequado & $83(73,5)$ \\
& Magreza & $3(2,7)$ \\
& Eutrofia & $76(67,3)$ \\
& Sobrepeso & $15(13,3)$ \\
& Obesidade & $15(13,3)$ \\
& Obesidade Grave & $4(3,5)$ \\
\hline
\end{tabular}

Tabela 2. Associação entre estado nutricional e fatores sociodemográficos e consumo alimentar de crianças e adolescentes matriculadas em escola pública de Ipiranga do Sul $(n=110)$.

\begin{tabular}{|c|c|c|c|c|c|c|}
\hline \multirow{3}{*}{ Variáveis } & \multirow{3}{*}{ Categorias } & \multicolumn{4}{|c|}{ IMC/idade* } & \multirow{3}{*}{ Valor de $p^{* *}$} \\
\hline & & \multicolumn{2}{|c|}{ Eutrofia } & \multicolumn{2}{|c|}{ Sobrepeso/obesidade } & \\
\hline & & $\mathbf{n}$ & $\%$ & $\mathbf{n}$ & $\%$ & \\
\hline \multirow[t]{2}{*}{ Sexo } & Masculino & 40 & 71,4 & 16 & 28,6 & \multirow{2}{*}{0,369} \\
\hline & Feminino & 36 & 66,7 & 18 & 33,3 & \\
\hline \multirow[t]{2}{*}{ Cor da pele } & Branco & 71 & 72,4 & 27 & 27,6 & \multirow{2}{*}{0,036} \\
\hline & Não branco & 5 & 41,7 & 7 & 58,3 & \\
\hline \multirow[t]{2}{*}{ Bolsa Família } & Sim & 4 & 33,3 & 8 & 66,7 & \multirow{2}{*}{0,008} \\
\hline & Não & 72 & 73,5 & 26 & 26,5 & \\
\hline \multirow[t]{2}{*}{ Classe Econômica } & Classes A e B & 44 & 77,2 & 13 & 22,8 & \multirow{2}{*}{0,044} \\
\hline & Classes C, D e E & 32 & 60,4 & 21 & 39,6 & \\
\hline \multirow[t]{2}{*}{ Consumo Alimentar } & Inadequado & 17 & 56,7 & 13 & 43,3 & \multirow{2}{*}{0,069} \\
\hline & Adequado & 59 & 73,8 & 21 & 26,3 & \\
\hline
\end{tabular}

* Foram excluídos os escolares com algum grau de magreza $(n=3)$.

** Teste Exato de Fisher. 
Tabela 3. Consumo alimentar de escolares matriculados em uma Escola Municipal de Ipiranga do Sul $(n=113)$

\begin{tabular}{lcccc}
\hline \multicolumn{1}{c}{ Alimentos } & $\begin{array}{c}\text { Nunca } \\
(\mathbf{\%})\end{array}$ & $\mathbf{1 - 2 x} \mathbf{( \% )}$ & $\mathbf{3 - 5 x} \mathbf{( \% )}$ & $\mathbf{> 6 \mathbf { 6 } ( \% )}$ \\
\hline Pães & 0,9 & 27,4 & 33,6 & 38,1 \\
Cereais e Grãos & 1,8 & 21,2 & 35,4 & 41,6 \\
Frutas & 8,8 & 33,6 & 34,5 & 23,0 \\
Verduras & 25,7 & 35,4 & 22,1 & 16,8 \\
Legumes & 23,9 & 51,3 & 19,5 & 5,3 \\
Leite & 7,1 & 13,3 & 33,6 & 46,0 \\
Peixes & 58,4 & 37,2 & 3,5 & 0,9 \\
Carnes & 13,3 & 19,5 & 38,1 & 29,2 \\
Carnes defumadas & 15,9 & 46,0 & 28,3 & 9,7 \\
Doces e petiscos & 5,3 & 40,7 & 37,2 & 16,8 \\
Refrigerante & 5,3 & 66,4 & 22,1 & 6,2 \\
Alimentos fritos & 11,5 & 54,9 & 30,1 & 3,5 \\
Alimentos grelhados & 17,7 & 51,3 & 21,2 & 9,7 \\
\hline
\end{tabular}

\section{DISCUSSÃO}

No presente estudo constatamos que os escolares do sexo masculino apresentam maior prevalência de excesso de peso de que o sexo feminino, o que se assemelha aos dados de Benedet et al. ${ }^{4}$, e difere dos resultados de Souza et al. ${ }^{6}$, o qual apresentou em seu estudo prevalência significativa de sobrepeso e obesidade no sexo feminino.

Quanto ao estado nutricional pode-se observar a prevalência de eutrofia, o que corrobora com estudo de Aires et al. ${ }^{14}$, realizado no Rio Grande do Sul, em uma escola pública de Santa Maria, utilizando a classificação pelo IMC, onde a maioria encontrou-se em eutrofia (66\%).

Já quanto ao perfil socioeconômico, observou-se predominância das classes B e C, o que difere do estudo de Rossi et al. ${ }^{15}$ que constatou prevalência das classes C (58,7\%), seguidas das classes B e D, já quanto ao recebimento de auxílios por parte do Estado, a maioria das famílias não os recebem o que corrobora com este estudo. Ainda de acordo com a avaliação do recebimento ou não do PBF, quando comparado com o presente estudo, foi possível observar que em estudo de Silva et al. ${ }^{16}$ foi encontrado percentual inferior (73\%), onde a maioria da amostra relataram não receber o benefício, e percentual superior (27\%) aos que recebem o benefício do PBF oferecido pelo governo federal.

$\mathrm{O}$ excesso de peso encontrou-se maior prevalência entre as classes econômicas mais baixas (C, D e E), o que difere do estudo de Rossi et al. ${ }^{15}$ que encontrou nas classes mais alta (B1 e B2), seguidas das medianas (C1 e C2) e menor prevalência do agravo entre uma das baixas classes econômicas (D).
Vale enfatizar que o presente estudo revelou uma maior frequência de excesso de peso entre os escolares que recebem o benefício do PBF. Este resultado é característico do fenômeno denominado "transição nutricional" que se caracteriza pela passagem da desnutrição para a obesidade, estando presente no Brasil e nos países em desenvolvimento, decorrente ao inadequado hábito alimentar ${ }^{17}$.

Em relação ao consumo alimentar constatamos que os alimentos com consumo adequado são os pães, cereais e grãos, leites e carnes. Assim como o estudo de Spinelli et al. ${ }^{18}$, o qual também apresentou o consumo adequado de cereais, massas, tubérculos e raízes, leite e derivados, porém apresentou consumo adequado de leguminosas e óleos e gorduras, os quais diferem de nosso estudo. Já os alimentos que apresentaram consumo inadequados são as frutas, verduras, legumes, peixes, carnes defumadas, doces e petiscos, refrigerantes, alimentos fritos e grelhados, o que corrobora com o estudo de Spinelli et al. ${ }^{18}$, ocorre o consumo inadequado de hortaliças, frutas, doces e açúcares e carnes.

Em relação ao consumo alimentar dos escolares estudados, os alimentos com consumo maior que 6 vezes na semana são os pães, cereais e grãos e o leite, este último, assemelha-se ao resultado de Levy et al. ${ }^{19}$. O estudo de Valente et al. ${ }^{20}$, analisou o consumo alimentar de escolares de uma creche da cidade de Santa Maria, e demonstrou que o leite e achocolatado em pó tem maior consumo $1 \mathrm{vez}$ ao dia. Em comparação o pão francês, biscoito recheado, macarrão, arroz branco, ovos, frango, banana, maçã, laranja, açúcar refinado, doces e refrigerantes tem maior consumo de 2 a 4 vezes na semana (>50\%). A carne bovina, espinafre, repolho e couve, cenoura e salgadinhos industrializados são consumidos com maior frequência de 1 a 4 vezes por mês e apenas os espessantes são consumidos raramente (76,9\%). Os dados diferem do presente estudo, pães, cereais e grãos são consumidos com frequência maior que 6 vezes na semana. Já em relação aos doces, carnes frutas, verduras, legumes e refrigerantes tem consumo de 1 a 2 vezes na semana, demonstrando que as crianças do nosso estudo apresentam uma alimentação mais adequada de que as do estudo de em Santa Maria.

O presente estudo apresenta como limitação o possível viés de resultados pelo fato de ser entregue o questionário de frequência alimentar para menores de 10 anos para que os responsáveis respondessem, os quais poderiam responder com alimentos que considerasse mais saudável e não que representasse a ingestão do escolar.

Desta forma, apesar do elevado percentual de eutrofia nos escolares estudados, destaca-se a prevalência de excesso de peso e alimentação inadequada. Este é um dos motivos que nos leva a implementar e articular estratégias locais e 
intersetoriais que possam contribuir para ações e praticas para a promoção da alimentação adequada e saudável com crianças e adolescentes deste município em diferentes cenários, como escolas, unidades de saúde e na comunidade.

\section{REFERÊNCIAS}

1. Souza EB. Transição Nutricional no Brasil: análise dos principais fatores. Cad UniFOA. 2010;13:49-53.

2. Vasconcelos IAL, Oliveira OMV. Panorama do estado antropométrico dos escolares brasileiros. Rev Paul Pediatr. 2011;29(1):108-16. https://doi.org/10.1590/S0103-05822011000100017

3. Batista Filho M, Leal VS, Lira PIC, Oliveira JC, Menezes Risia CE, Sequeira LAS. Excesso de peso em crianças e adolescentes no Estado de Pernambuco, Brasil: prevalência e determinantes. Cad Saúde Pública. 2012;28(6):1175-82. https://doi.org/10.1590/S0102311X2012000600016

4. Benedet J, Assis MAA, Calvo MCM, Andrade DF. Excesso de peso em adolescentes: explorando potenciais fatores de risco. Rev Paul Pediatr. 2013;31(2):172-81. https://doi.org/10.1590/S010305822013000200007

5. Ministério do Planejamento, Orçamento e Gestão (BR); Instituto Brasileiro de Geografia e Estatística-IBGE. Pesquisa de Orçamentos familiares 2008-2009: Antropometria e Estado Nutricional de Crianças, Adolescentes e Adultos no Brasil. Brasília: Ministério do Planejamento Orçamento e Gestão; 2010.

6. Souza TRF, Araújo RA. Perfil Antropométrico em escolares da rede pública. Ed. Física em Rev. 2011;5(1):1-8. https://doi.org/10.1590/ S1806-11172011000100026

7. Ministério da Saúde (BR). Secretaria de Atenção à Saúde. Departamento de Atenção Básica. Política Nacional de Alimentação e Nutrição. Brasília: Ministério da Saúde; 2012.

8. Câmara Interministerial de Segurança Alimentar e Nutricional. Estratégia Intersetorial de Prevenção e Controle da Obesidade: recomendações para estados e municípios. Brasília, DF: CAISAN; 2014.

9. Ministério da Educação (BR). Fundo Nacional de Desenvolvimento da Educação (FNDE). Conselho Nacional dos Procuradores Gerais do Ministério Público dos Estados, do Distrito Federal e da União. Grupo Nacional de Direitos Humanos. Cartilha Nacional da Alimentação Escolar. Brasília, DF: Ministério da Educação; 2015.
10. Associação Brasileira de Empresas e Pesquisas. Critério de Classificação Econômica Brasil. São Paulo: ABEP; 2015.

11. Ministério da Saúde (BR). Norma Técnica do Sistema de Vigilância Alimentar e Nutricional-SISVAN. Orientações para a coleta e análise de dados antropométricos em serviços de saúde. Brasília: Ministério da Saúde; 2011.

12. Frisancho AR. Anthropometric standards for the assessments of growth and nutritional status. Ann Arbor: University of Michigan Press; 1990. https://doi.org/10.3998/mpub.12198

13. Martins C. Avaliação do Estado Nutricional de Crianças e Adolescentes. Curitiba: Instituto Cristina Martins; 2009.

14. Aires AP, Botega AO, Pedron F, Pinto G, Ramos N, Pereira P, Saccol ALF. Perfil nutricional de alunos em escola pública. Disc Scientia Série Cienc Saúde. 2009;10(1):77-86.

15. Rossi CE, Machado AD, Piano CM, Beal GK, Cavaller SC, Zolet T, Lazarotto AK, Pizzattol CC. Perfil antropométrico e socioeconômico de escolares da rede pública de ensino de município paranaense. G\&S. 2015;6(2):1463-76.

16. Silva MC, Capanema FD, Lamounier JA, Silva ACA, Oliveira BM, Rodrigues J. Perfil nutricional de crianças pré-escolares em creches públicas de Belo Horizonte - Minas Gerais beneficiárias ou não do Programa Bolsa Família. Perc Acad. 2014;4(7):88-104.

17. Souza CPC, Sousa MPC, Rocha ACD, Pedraza DF. Perfil epidemiológico do estado nutricional de crianças assistidas em creches no Estado da Paraíba. Rev. Soc. Bras. Alim. 2011;36(1):111-26.

18. Spinelli MGN, Morimoto JM, Freitas APG, Barros CM, Dias DHS, Pioltine MB, Gonçalves PPO, Navarro RB. Estado Nutricional e Consumo Alimentar de pré-escolares e escolares de escola privada. Ciênc Saúde. 2013;6(2):94-101. https://doi.org/10.15448/1983$652 X .2013 .2 .12686$

19. Levy RB, Castro IRR, Cardoso LO, Tavares LF, Sardinha LMV, Gomes FS, Costa AWN. Consumo e comportamento alimentar entre adolescentes brasileiros: Pesquisa Nacional de Saúde do Escolar (PeNSE), 2009. Ciênc Saúde Coletiva. 2010;15(2):3085-97. https:// doi.org/10.1590/S1413-81232010000800013

20. Valente TB, Hecktheuer LHR, Brasil CCB. Condições socioeconômicas, consumo alimentar e estado nutricional de préescolares pertencentes a uma creche. Alim Nutr. 2010;21(3): 421-8. 